\title{
A proposed Estimation for High Performance Level of VVoIP System on a live Service IMS Network
}

\author{
Hanafy M. Ali ${ }^{1}$, Waled M Ismail ${ }^{2}$, Aziza I. Hussein ${ }^{3}$, Yahia B.Hassan ${ }^{4}$ \\ 1Computers and Systems Engineering Depart., -Faculty of Engineering, Minia University, El Minia, Egypt, hmali@mu.edu.eg \\ 2 Mobile core back office engineer, Telecom Egypt CO. , Egypt, Waleed.mahamad@te.eg, \\ 3 Electrical and Computer Eng. Dept. Effat University, Jeddah, Saudi Arabia, azibrahim@effatuniversity.edu.sa , \\ 4Electrical Eng. Dept, Higher Institute of Engineering, Minia, Egypt, dryahiabahaahassan@ gmail.com
}

\begin{abstract}
In the last few years, the use of Video and Voice Over Internet Protocol (VVOIP) has grown rapidly due to its cost effectiveness and dramatic functionality over the traditional telephone network. Reliance on VVOIP applications like video conferences, video calls and live videos has become imperative recently since following social-distancing guidelines as a result of the widespread of COVID-19 pandemic. In spite of the increase in the internet traffic and the limitation of bandwidth, the customers always need more interactive multimedia applications with high quality and efficiency. Hence, the quality of delivery for VVoIP applications should be measured and optimized to ensure Quality of Service (QoS) and Quality of Experience (QoE) support to customers in real time video call systems. In this paper, video-related QoE metrics like quantization parameter (QP), frame rate (FR) and resolution are implemented for quality evaluation of a video call over live service network based on IP multimedia subsystem (IMS). Furthermore, this paper investigates the effect of bandwidth limitation on network-related QoS parameters such as packet loss and jitter and then on video calls QoE on a live network based on IMS by using a Wireshark 3.0.0 software as protocol analyzer to define QoE and QoS metrics and to determine the best performance of VVoIP systems.
\end{abstract}

Keywords: VOIP, VVOIP, QoE, QoS, IMS.

\section{INTRODUCTION}

Voice over Internet Protocol (VOIP) is very popular in recent days and has become the first choice for national to multinational companies for the integration of voice and data in order to reduce the cost and to optimize the utilization of network resources [1].

The spread of VOIP applications is not limited to wired networks only, but also via mobile network technologies.

As the high-speed mobile network has been widely adopted, the smart devices enable users to achieve high-quality and high data rate communication VOIP for the mobile environments such as Google+, Hangouts, Skype, WeChat and Line [2].

In the last three decades, communication technology has been developed tremendously. Starting from limited services mobile cellular systems and Signaling System No. 7 (SS7) based networks to the completely IP networks with multimedia services and multi access [3].

The inevitability of a unified core for all access networks has increased to support communication services between users independent of the access networks such as landline, mobile, IP television, IP phone, etc. Third generation partnership project (3GPP) has been introduced IMS as unique core, based on IP for all access networks. Few years later, the dependence on IMS has become the trend for all

Revised:14 July, 2021, Accepted:6 Novamber, 2021 local and international telecommunication operators.

One of the most important applications based on IMS is VVOIP such as Video conferences, e-learning live video streaming which allows the communication by voice and video in real time communication channel.

Users want to share high-quality videos independent of their internet connection and available bit rate. By 2021, every second a million of video minutes will cross the Internet. Video streaming is responsible for more than $80 \%$ of all the IP traffic [4]. To ensure high-quality video calls, many parameters must be optimized specially in the limitation of network available bit rate. This paper investigates the effect of QOE parameters of the video call under live service IMS network on the consumed bit rate during the call. The most effective QoE parameters are video resolution, Quantization Parameter and frame rate [5] while the most affecting parameters on Video call quality are the packet loss and the variation in the delay of the received packets (Jitter). [6] [7].

Since bandwidth consumption is the main challenge in IP networks, this paper also examines the effect of bandwidth limitation on QoS parameters (packet loss and mean jitters) and video calls (QoE). The common factor that controls both packet loss and jitter is the available network bandwidth which is affected by quantization parameter, frame rate and resolution. In the first phase of the study, a live traffic network based on IMS has been used to test the impact of quantization parameter, frame rate and video 
resolution on the consumed data rate. As a result, we can get the optimum values of the resolution, frame rate and quantization parameter for a given network available bandwidth.

The effect of bandwidth restriction on packet loss and jitter has been tested in the second phase to get accepted values of packet loss and jitter. The estimation of packet loss and jitter in low bandwidth networks will support in defining the suitable video parameters in order to enhance video call quality.

\section{OVERVIEW OF VVOIP AND IMS}

VVoIP refers to the technologies, protocols and transmission techniques involved in the communication of video and voice (multimedia sessions) over Internet Protocol (IP) based networks, such as the Internet.

The steps involved in originating a VVoIP call are signaling and media channel setup, digitization of the analog signal, encoding, packetization, and transmission as IP packets over a packet-switched network. On the receiving side, similar steps (usually in the reverse order) such as reception of the IP packets, decoding of the packets and digitalto-analog conversion reproduce the original voice or video stream as shown in fig. (1) [8].

VVoIP applications/clients are available on many platforms such as smart phones, personal computers and Internet devices.

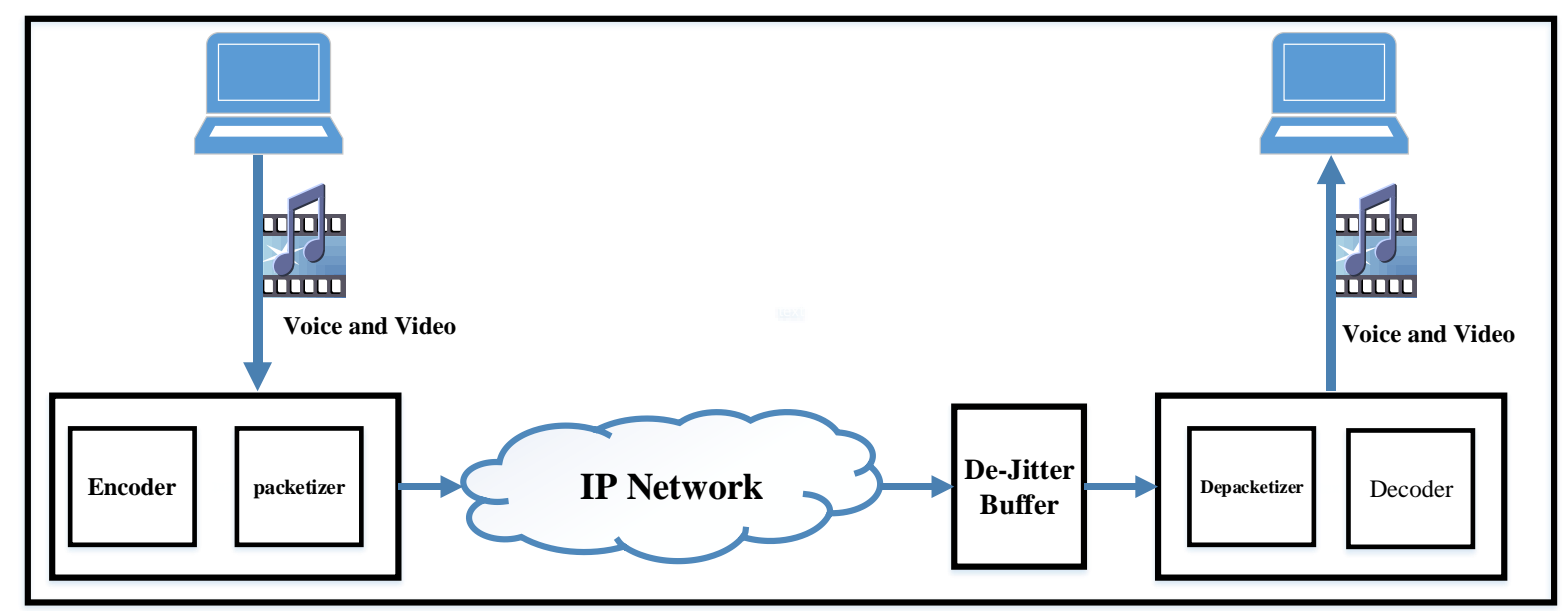

Fig. 1. High-level VVoIP System Architecture

As VVOIP applications become more important and the users become more dependent on them, the communication service providers need more network integrity and access independent which is granted by IMS.

IMS is a combination of functional elements (nodes) connected with standard interfaces which act as network core independent of access networks via session initiation protocol (SIP). As a signaling protocol for real-time communication, SIP performs user location, session establishment, and session management [9] SIP is the most efficient used signaling protocol because of it's text-based structure and also end to end connectivity, supports mobility, and is independent from transferred data type [10]

The architecture of IMS is based on a functional element called Call Session Control Function (CSCF) where it is the main part of IMS which is used to setup, maintain and release call sessions [11]. CSCF also handles the quality of service control as the session control function includes codec selection and negotiation. Other functions are also based on CSCF like subscriber registration, authentication, billing and deregistration. From the functionality side, CSCF consists of three sub nodes: Proxy CSCF, Servicing CSCF and Interrogating $\mathrm{CSCF}$.

1) The Proxy Call Session Control Function: (P- 
CSCF) is the entry point for the IMS user. Signaling traffic from User Equipment (UE) to the core as well as from any SIP node is basing through the P-CSCF to the UE. In other words, all requests and responses initially go through P-CSCF. In addition to that, P-CSCF analyze the request from $\mathrm{UE}$ to define the needed service and which S-CSCF can handle this service.

2) The Serving Call Session Control Function: (S-CSCF) has some functions related to the service handling and service control as it is responsible for user registration. S-CSCF receives the registration request from the user and responds to it depending on the user profile in the Home Subscriber Server (HSS). Session control is based on the available session parameters and user request. It is also responsible for session termination and billing records. On the other hand, it is responsible for the communication with the application server in the application layer in case of demand as per the analysis of user request.
3) The Interrogating Call Session Control Function: (I-CSCF) is responsible for the communication with other networks. Any request from other network passes through the I-CSCF. In the roaming scenario I-CSCF handles the registration and selection of $\mathrm{S}$ CSCF function.

In addition to the CSCF, HSS (based on Diameter Protocol) is the master user database that supports the IMS network entities that handle the call sessions. HSS contains the subscription-related information (user profiles), used by the control layer. Also, it contains subscription information used by the service layer and provides data used to perform authentication and authorization of the user. Moreover, HSS can provide information about the physical location of user. Fig (2) indicates the proposed model architecture of VVOIP network based on IMS connected to session boarder controller (SBC) to establish a session for video and data exchange between two clients.

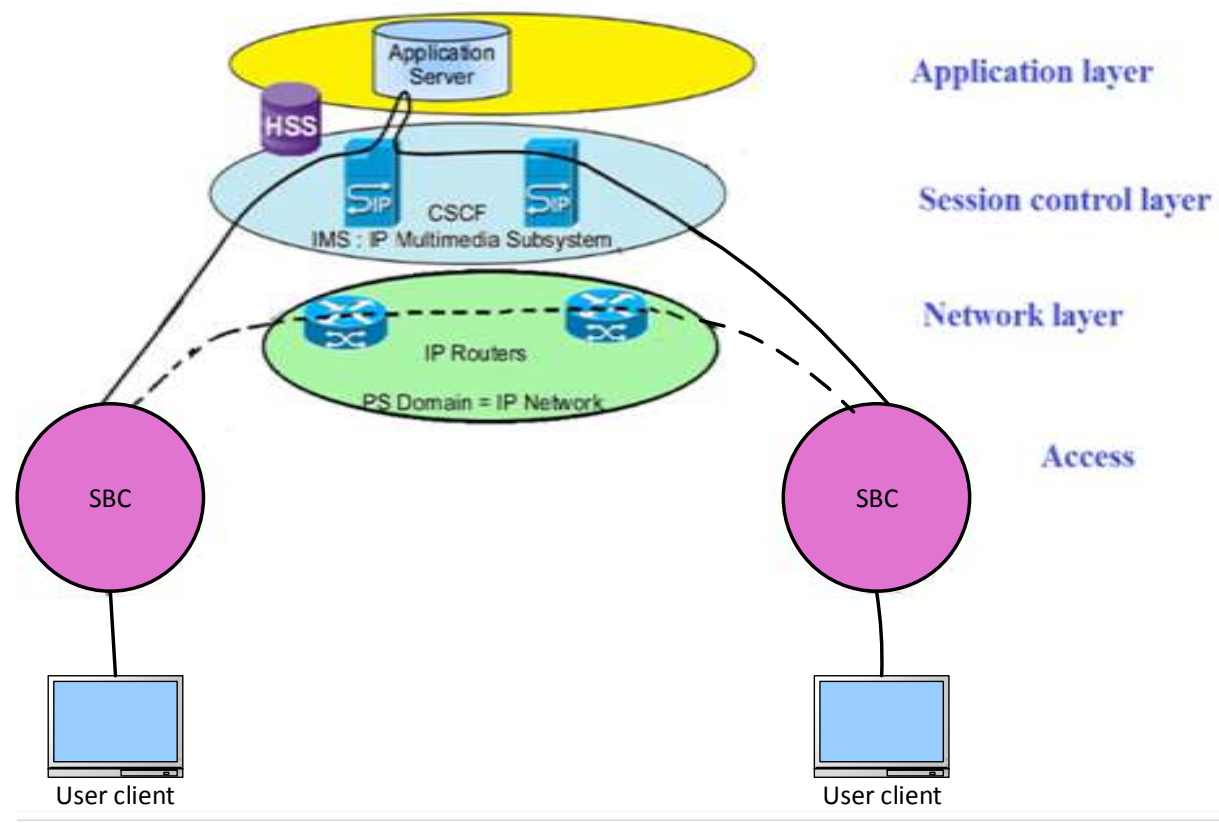

Fig.2. the proposed VVOIP model based on IMS network

A number of messages will be exchanged to establish, control and terminate this session. Fig 3 presents the call flow of VVOIP session establishment between client A and client B based on system model in fig (2). Seven steps to achieve VVOIP call flow between two clients should be performed as follows:
(1) Subscriber A initiates a video sharing session request to subscriber B through an INVITE message. After receiving the request, subscriber $\mathrm{B}$ returns a 200 OK message.

(2) Subscriber B intercepts and binds the IP address and port number carried in the Session Description 
protocol (SDP) of the 200 message and sends a Realtime Transport Protocol (RTP) connection request to the IP address and port number carried in the SDP of the received INVITE message.

(3) Subscriber A binds the IP address and port number carried in the SDP of the sent INVITE message and sends an RTP/ Real-time Transport control Protocol (RTCP) connection request to the IP address and port number carried in the SDP of the received 200 messages.

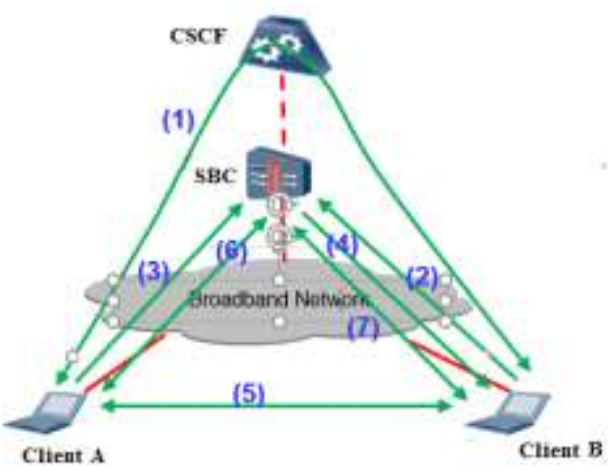

Fig.3. VVOIP call flow

(4) The RTP or RTCP packet sent by subscriber A successfully arrives at subscriber B. Then, a connection is successfully established between the two subscribers.

(5) Subscriber A sends video information to subscriber B through the RTP protocol.

(6) The party terminating the sharing must send a $B Y E$ request to the other party and then terminates the RTP flow.

(7) The other party responds to the BYE request by 200 OK message.

\section{RELATED WORK}

A large portion of the researches in VVOIP QoE has focused on the study of the individual effect of network parameters on the QoS using simulation models. The authors [ 12] have investigated the impact of impaired network and packet loss on QoE of encoded video streams. The quality of the video conferencing was assessed by subjective tests [13] and [14]. The use of VVOIP network emulation to estimate VVOIP call has been introduced in [ 8]

By using software over internet, the impact of network limitation on one participant of multiparty conference call to the total end users QoE had been studied in [ 15] by controlling three network parameters which are layout, video resolution and network limitation. The study results have showed the significant effect of packet loss and video resolution on end user QoE. The study had also showed that the end users were more satisfied when the network layout and limitation were similar.

Where in [5], the authors have proposed a prediction model to predict the video QoE based on two main types of parameters named as network layer QoS and application layer QoS and tried to find a relation between the network layer QoS parameters and the Video call QoE parameters using the designed model. The assessment of subjective video of full HD resolution has been discussed in [16].

Factors affecting the quality of the experience for video calls have been studied in [ 17], while the impact of network bandwidth limitation on quality of video conferences has been presented in [18]. In [ 5], D. Vucic and L. Skorin-Kapov have investigated how different video encoding parameters in terms of encoding bit rate, video resolution and frame rate influence QoE. The effect of packet loss on QoE audio and video call is shown in [19].

The main contribution of this paper is to study the impact of resolution, Q.P. and F.R. on the consumed bitrate of the video call and the network bandwidth. This study has been applied on a proposed model based on live IMS network at Almaza region which controls service all over Egypt. So, this study is very important for practical networks designers and operators. In addition, this paper investigates the impact of bandwidth restriction on the packet loss, jitter and the video call quality.

\section{QOE AND QOS EVALUATION OF VVOIP SYSTEM}

The dependency of users on VVOIP applications increased strongly. According to OWL Labs [ 20], one of the major companies that creates video conferencing equipment, in 2019 identified that there were $52 \%$ of US-based employees working from home before the social distancing. This enhanced the operators to investigate in the parameters affecting the QoE and the correlation between network QoS related parameters (bandwidth, packet loss and Jitter), video related metrics and QoE

The correlation between QoS parameters and video QoE parameters is very useful to define the impact of network QoS on video QoE and to get an accurate estimation for high performance level of VVoIP system. This paper has investigated in the impact of QoE parameters on the consumed bandwidth by capturing the flow 
of signaling through the network and analyzing it to extract conclusions. This method is characterized as being suitable for different sizes of networks, as it is possible in all cases to capture and analyze signals. It is also scalable, as the analysis can include a larger number of variables Trying to find the best scenario to improve the video quality on a live service IMS network with dynamic change in bandwidth has been presented in this paper.

\section{A. Software}

Wireshark 3.0.0 program has been used for our system model as open source for packet tracing and protocol analyzer. $\mathrm{s}$. This software has been installed under Windows 7 on core I 5 HP laptop 2.7 Ghz.

B. SYSTEM MODEL

In this research we have performed our investigations on a large scale live network based on IMS. It provides voice and video calls services in addition to data services for several millions of users all over Egypt, whether fixed or mobile, through Ericsson IMS in Cairo. The architecture of VVOIP live network is based on IMS connected to (SBC) via Ethernet cable and cisco switch to user equipment as shown in Fig. 2 . The user equipment in our experiment is Cisco DX 80 video conference equipment with 23 -inch $(0.58 \mathrm{~m})$ LCD monitor, resolution: 1920 x 1080 (16:9), Front video camera of $30 \mathrm{fps}$, full HD resolution under Android operating system with $1.5-\mathrm{GHz}$ dual-core ARM Cortex-A9 processor and 2-GB RAM. Throughout the experiment, the network key performance indicators (KPIs) have been monitored closely to ensure that all network parameters are stable during the experiment and to ensure that results are not dependent on network performance variation. The following KPIs were monitored during the experiments:

- IMS Session Success Ratio

- IMS CSCF Terminating Session Set-up Success Ratio

- IMS CSCF Originating Session Set-up Success Ratio

- IMS CSCF Originating Sessions Set-up Time [ms]

- IMS CSCF Terminating Sessions Set-up Time [ms]

- IP QoS Discard Rate

- Media Stream resource utilization ratio

- RTP Packet Discard Rate

- RTP Packet Loss Rate
The two phases implemented for the system model being studied are as follows:

\section{Phase 1: The effect of QoE parameters on bitrate}

In the first phase of this study, the effect of three QoS parameters on the bit rate of the live network video call has been investigated, the three parameters are:

i) Video resolution: The most widely used resolution values in video conferencing now $(480 \mathrm{p}, 720 \mathrm{p}$, and 1080p) have been used.

ii) Frame rate in frame per second (fps): We have selected frame rates $20 \mathrm{fps}$, $25 \mathrm{fps}$ and $30 \mathrm{fps}$.

iii) Compression ratio or Quantization parameter (QP): the following QPs are considered 20, 24,28,32,36 and 40 .

Phase 2: The effect of bandwidth restriction on $Q o S$ parameters

In this phase of the study, the effect of bandwidth restrictions on the most common QoS parameters has been implemented to show the effect of network congestion on video call. The considered QoS parameters are:

i) Percentage packet loss.

It means the percentage of lost packets which were not received at $B$ side to the total sent packets at A side.

ii) Mean jitter in ms.

Jitter is defined as the variation in the delay of packets arrival at the destination. The main impacts of jitter buffer are latency and packet loss.

\section{RESULTS AND DISCUSSION}

A. Phase 1 results

i) For video calls at $480 \mathrm{p}$

Fig 4. Shows the effect of frame rate and quantization parameter on consumed bitrate at resolution of $480 \mathrm{p}$. The results generally have showed a decrease in the consumed bit rate with the increase in the Q.P. for all frame rates because the Q.P. reflects the compression in the transmitted data. It is important to mention that the consumed bit rate is not decreasing in the same rate for all QPs used as the consumed bit rate decreases sharply by increasing the QP from 20 to 28. Also, the difference between consumed bit rate for the three frame rates decreases. Starting from QP equal 32, we could observe that the impact of increase in the QP was very small in 
Vol.42, No.1. January2023

comparison to smaller QPs. also the difference between bit rates for three frame rates were not observed. This means that the sharpness of the bit rate curve decreased by increasing the QP starting from 28 to 40. The difference between the consumed bit rate for the used frame rates decreased by increasing the QP which means that the three curves are closer to congruence starting from point 32 . This reflects that the increment in QP greater than 32 will not reflect significant saving in BW.

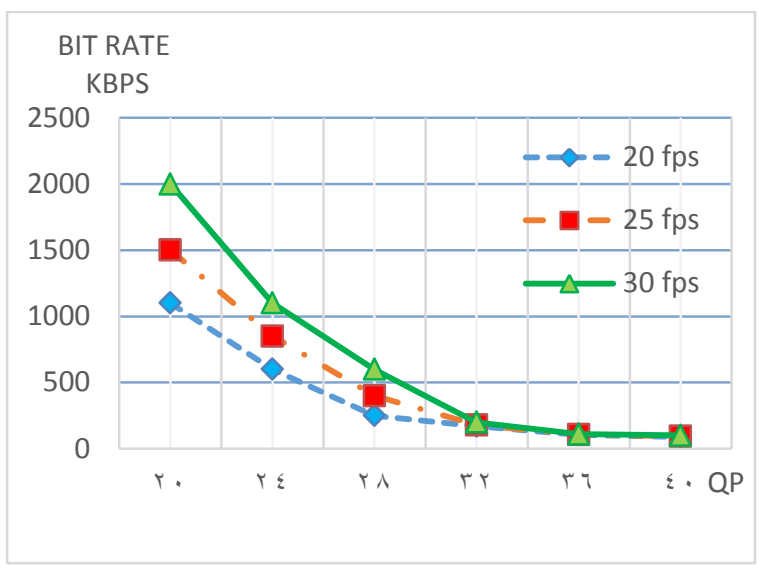

Fig.4. Bit rate versus QP at frame rates ( 20, 25 and $30 \mathrm{fps}$ ) for resolution $480 \mathrm{p}$

i) For video calls at $H D(720 p)$

Fig.5 presents the effect of QP on consumed bit rate at HD. It is clear that the consumed bit rate increased for HD resolution in comparison with the $480 \mathrm{p}$ and the consumed bit rate decreased by the increment in the QP. In the next results, the rate of variation in the consumed bit rate by the change in the QP has been considered. the results have indicated that there was a sharp decrease in consumed bit rate by increasing the QP from 20 to 28 while this sharpness degraded at QP from 28 to 32. At QP from 32 to 40, the rate consumed bit rate decreased and there was a flat curve in this range. Regarding the difference in consumed bit rate between the three frame rates, we could observe that the difference decreased gradually by increasing the QP till QP equal 32. At QP from 32 to 40, there was a slight difference in the consumed bit rate between the three frame rates at 20fp, 25fps and 30fp.This means that the curve in HD will proceed in sharp decrease of consumed bit rate vs. increasing QP at QP equal 28 .

The previous resolution showed a congruence in the three frame rate curves at QP 32. The HD resolution showed an observed difference in the bit rates at point 32 , while the curves appear identical at point 36

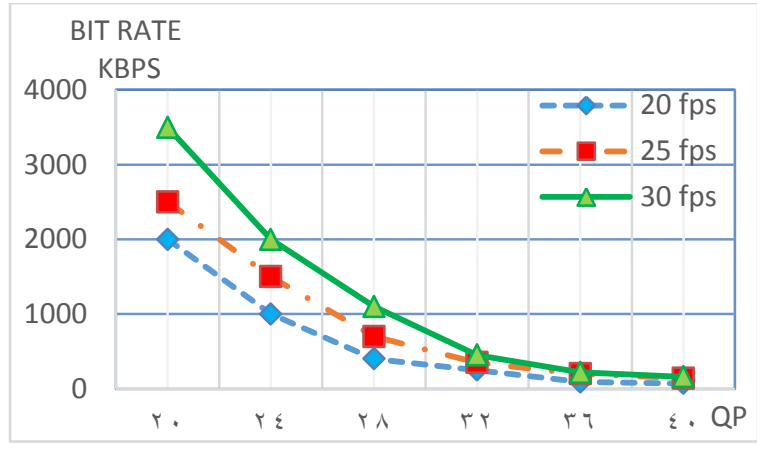

Fig.5. Bit rate versus QP at frame rates ( 20, 25 and $30 \mathrm{fps}$ ) for HD resolution

\section{ii) For video calls at full HD (1080p)}

In this resolution, the consumed bitrate had increased to $4000,5000,6000 \mathrm{Kbps}$ approximately at frame rates 20, 25 and $30 \mathrm{fps}$ respectively. Also, the impact of QP remained sharp even after QP equal 32 which indicates the effect of QP on BW consumption was the greatest at this resolution as shown in Fig.6. Moreover in this resolution, the differences in the consumed bit rate between the three frame rates had increased in comparison with other resolutions even at high QP. This indicates that the impact of frame rate on the consumed bit rate is greater at higher resolutions.

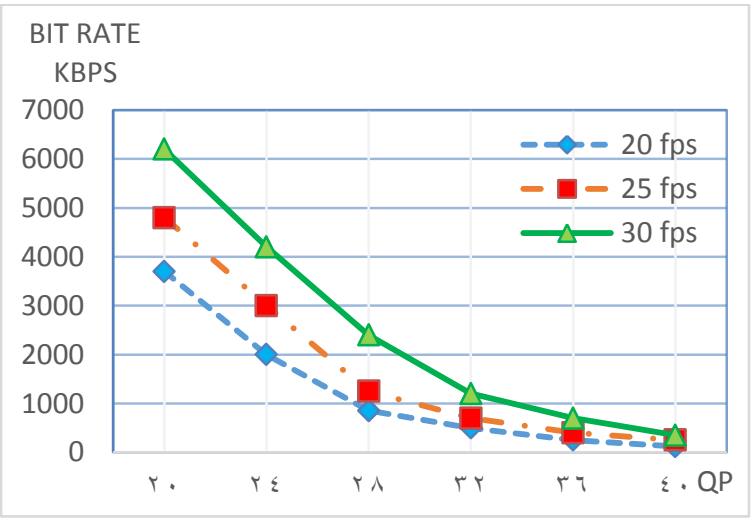

Fig. 6: Bit rate versus QP at frame rates ( 20, 25 and $30 \mathrm{fps}$ ) for Full HD resolution

\section{B. Phase 2 results}

In this phase, the impact of the available data rate on packet loss and jitter of video call for different video resolutions had been studied to obtain the best possible QoE available to the end user. We had simulated the life network performance at the leakage of available bit rate especially at high traffic condition. The simulation had been done by restricting the available network bit rate to five different bit rate values to study the impact of that leakage on packet loss and jitter. 
For the three given video resolution $480 \mathrm{p}, 720 \mathrm{p}$ and $1080 \mathrm{p}$, we had observed the percentage packet loss and mean jitter on video call under restricted network bit rates of $1200 \mathrm{kbps}, 1000 \mathrm{kbps}, 800 \mathrm{kbps}, 600$ kbps and $400 \mathrm{kbps}$. The bit rate restriction had been applied by controlling the bit rate stream on the juniper router connected to the cisco video client. At each restriction stage, a data stream of $5000 \mathrm{kpbs}$ was exchanged to confirm that bit rate restriction is applied by using Wireshark client. Results of packet loss and mean jitter had been obtained by analyzing 150 video calls (10 calls for each of the five restriction levels according to the three mentioned resolutions) and duration of each call is 120 seconds.

The average of packet loss and jitter for 150 calls is shown in table1.

Table 1: percentage packet loss and mean jitter at bit rate restriction

\begin{tabular}{|c|c|c|c|}
\hline \multirow{4}{*}{ Resolution } & $\begin{array}{c}\text { Bit } \\
\text { rate } \\
\text { kbps }\end{array}$ & $\begin{array}{c}\text { Packet Loss } \\
\%\end{array}$ & $\begin{array}{c}\text { Mean } \\
\text { jitter ms }\end{array}$ \\
\hline \multirow{4}{*}{ 480 P } & 1200 & 0.12 & 6.5 \\
\cline { 2 - 4 } & 1000 & 0.19 & 7.4 \\
\cline { 2 - 4 } & 800 & 0.27 & 8.2 \\
\cline { 2 - 4 } & 600 & 0.31 & 8.8 \\
\cline { 2 - 4 } & 400 & 0.36 & 9 \\
\hline \multirow{4}{*}{$\mathbf{7 2 0} \mathbf{P}$} & 1200 & 0.18 & 8.7 \\
\cline { 2 - 4 } & 1000 & 0.21 & 9.1 \\
\cline { 2 - 4 } & 800 & 0.28 & 10.5 \\
\cline { 2 - 4 } & 600 & 0.47 & 11.2 \\
\cline { 2 - 4 } & 400 & 0.61 & 12 \\
\hline \multirow{5}{*}{$\mathbf{1 0 8 0} \mathbf{P}$} & 1200 & 0.28 & 11 \\
\cline { 2 - 4 } & 1000 & 0.34 & 11.8 \\
\cline { 2 - 4 } & 800 & 0.4 & 12.5 \\
\cline { 2 - 4 } & 600 & 0.72 & 15.3 \\
\cline { 2 - 4 } & 400 & 0.95 & 18.7 \\
\hline
\end{tabular}

\section{For percentage packet loss}

It is demonstrated in Fig. 7 that there is a convergence in results somewhat for $1080 \mathrm{p}, 720 \mathrm{p}$ and $480 \mathrm{p}$ resolutions at the bit rates especially for both 480 and 720p where the packet loss percentage appears to be nearly identical from $1200 \mathrm{kbps}$ to 800 kbps. By restricting network bit rate from 600 to $400 \mathrm{kpbs}$, the extreme difference between the three resolutions is clearly observed as the packet loss curve increased sharply for both $720 \mathrm{p}$ and $1080 \mathrm{p}$ while the curve of $480 \mathrm{p}$ keeps the same rate of increase.

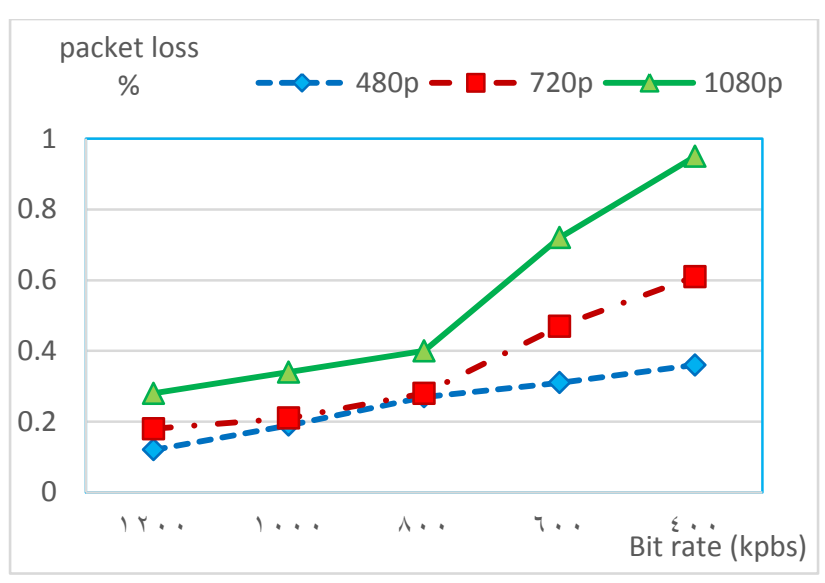

Fig. 7: percentage packet loss at restricted bit rates for 480P, 720P and $1080 \mathrm{P}$

\section{ii) For mean jitter}

Close examination of Fig. 8 reveals that at both 480p and $720 \mathrm{p}$ resolutions, we can observe that the smooth rate of average jitter increases by restricting the network bit rate for all values of bit rates. While the average jitter curve at $1080 \mathrm{p}$ increases with the same rate for bit rates from $1200 \mathrm{kpbs}$ to $800 \mathrm{kpbs}$ and for further bit rate restriction, the mean jitter increases sharply reaching more than $18 \mathrm{msec}$ at $400 \mathrm{kpbs}$.

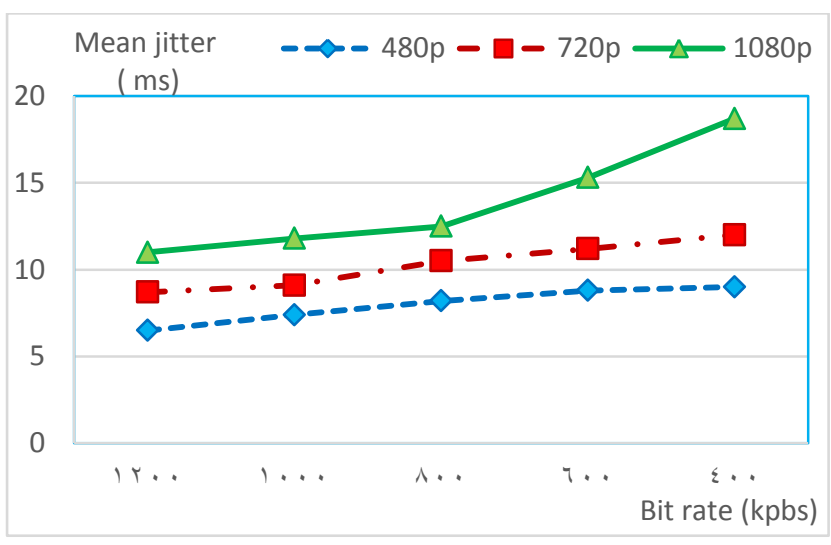

Fig. 8: Mean jitter at restricted bit rates for 480P, 720P and 1080P

\section{CONCLUSION}

By increasing the spread of life video dependent applications like video calls, video conference and video games, the demand of high bit rate data links increased as these applications are the most bit rate consuming. In this paper, a framework for studying the effect of different QoE and QoS VVOIP parameters on the performance of VVoIP systems has been introduced. Unlike other similar studies, we have specifically focused to make an implementation 
for performance estimation of a video call over live service network based on IMS.

The aim of the first stage experiment is to verify the effect of QoE parameters of the video on the consumed bit rate. We have examined the effect of video resolution (480p, 720p and 1080p), quantization parameters (20 up to 40 ) and three frame rates of $20 \mathrm{fps}, 25 \mathrm{fps}$ and $30 \mathrm{fps}$ on the consumed bit rate of the video call.

From the results of the first phase, we can infer the degradation rate of the consumed bit rate by increasing the QP. We have also determined the variance points in the rate which named as transaction points in QP.

In phase 2, we have investigated the impact of bit rate restriction on QoS parameters of the video like packet loss and mean jitter and also on QoE for the three resolutions 480p, 720p and 1080p. The restriction in bit rate has been implemented by restricting the available network bit rate to five different levels (400 kpbs, $600 \mathrm{kpbs}, 800 \mathrm{kpbs}$, $1000 \mathrm{kpbs}$ and $1200 \mathrm{kpbs}$ ) and at each restriction level, we have defined the percentage packet loss and mean jitter. By analyzing the results of phase 2, it has concluded that there is an increase in the packet loss rate and mean jitter when the bit rate is restricted for the three resolutions. The results indicate that $\mathrm{QoE}$ will be affected severely at limited bandwidth especially at high resolution.

\section{REFERENCES.}

[1] Zahid Ali, Falak Naz, Javed,Muhammad Qurban, Muhammad Yasir, Shehroz Jehangir,"Analysis of VoIP over Wired \& Wireless Network with Implementation of QoS CBWFQ \& 802.11e", International Journal of Computer Network and Information Security(IJCNIS), Vol.12, No.1, 2020, pp.43-49. DOI:10.5815/ijcnis.2020.01.05

[2] J. P. Singh, M. K. Mishra and M. A. Khan, "Energy-efficient approach towards video-based sensor networks (wireless) beneath barrier coverage," 2017 8th International Conference on Computing, Communication and Networking Technologies (ICCCNT), Delhi, 2017, pp. 15.doi: 10.1109/ICCCNT.2017.8204143

[3] S. Daoud and Y. Qu, "A Comparison Research on DSCP Marking's Impact to the QoS of VoIPbased and SS7-based Phone Calls," 2019 7th International Conference on Information, Communication and Networks (ICICN), Macao, Macao, 2019, pp. 66-71. doi: 10.1109/ICICN.2019.8834943

[4] B. U. Maheswari and T. K. Ramesh, "An Improved Delay-Resistant and Reliable Hybrid Overlay for Peer-to-Peer Video Streaming in Wired and Wireless Networks," in IEEE Access, vol. 6, 2018, pp. 56539-56550. doi: 10.1109/ACCESS.2018.2871932
[5] D. Vucic and L. Skorin-Kapov, "QoE evaluation of WebRTC-based Mobile Multiparty Video Calls in Light of Different Video Codec Settings," 2019 15th International Conference on Telecommunications (ConTEL), Graz, Austria, 2019, pp. 1-8, doi: 10.1109/ConTEL.2019.8848499.

[6] Moufida Maimour, SenseVid : A traffic trace based tool for QoE Video transmission assessment dedicated to Wireless Video Sensor Networks, Simulation Modelling Practice and Theory, Volume 87, 2018, Pages 120-137, ISSN 1569-190X https://doi.org/10.1016/j.simpat.2018.06.006

[7]Yudha, Dandun Kusuma, and Ahmad Ashari. "Analysis of Video CODEC Performance Using Different Softphone Applications." 2021 IJCCS (Indonesian Journal of Computing and Cybernetics Systems) 15.2: 153-164.

[8] J. Nightingale, Q. Wang, C. Grecos and S. Goma, "The impact of network impairment on quality of experience (QoE) in H.265/HEVC video streaming," in IEEE Transactions on Consumer Electronics, vol. 60, no. 2, May 2014, pp. 242 250. doi: 10.1109/TCE.2014.6852000.

[9]A. Montazerolghaem, M. H. Y. Moghaddam and A. Leon-Garcia, "Open SIP: Toward SoftwareDefined SIP Networking," in IEEE Transactions on Network and Service Management, vol. 15, no. 1, pp. 184-199, March 2018, doi: 10.1109/TNSM.2017.2741258.

[10]A. Montazerolghaem, S. -. Shekofteh, G Khojaste, M. Naghibzadeh and M. Yaghmaee$\mathrm{M}$, "A novel load scheduling for session initiation protocol networks," 2014 4th International Conference on Computer and Knowledge Engineering (ICCKE), 2014, pp. 509-514, doi: 10.1109/ICCKE.2014.6993376

[11]A. Montazerolghaem, M. H. Yaghmaee, A. LeonGarcia, M. Naghibzadeh and F. Tashtarian, "A Load-Balanced Call Admission Controller for IMS Cloud Computing," in IEEE Transactions on Network and Service Management, vol. 13, no. 4, pp. 806-822, Dec. 2016, doi: 10.1109/TNSM.2016.2572161

[12] G. Berndtsson, M. Folkesson, and V. Kulyk, "Subjective quality assessment of video conferences and telemeetings," in Packet Video Workshop (PV), 2012 19th International, 2012, pp. 25-30.

[13] M. Ndiaye, M. C. Larabi, H. Saadane, G. L. Lay, C. Perrine, C. Quinquis, and L. Gros, "Subjective assessment of the perceived quality of video calling services over a real LTE/4G network," in 2015 Seventh International Workshop on Quality of Multimedia Experience (QoMEX), 2015, pp. 1-6.

[14] H. Assem, M. Adel, B. Jennings, D. Malone, J. Dunne and P. O'Sullivan, "Online estimation of VVoIP Quality-of-Experience via network emulation," 24th IET Irish Signals and Systems Conference (ISSC 2013), Letterkenny, 2013, pp. 1-8, doi: 10.1049/ic.2013.0031

[15] M. Schmitt, J. Redi, P. Cesar and D. Bulterman, "1Mbps is enough: Video quality and individual idiosyncrasies in multiparty HD videoconferencing," 2016 Eighth International Conference on Quality of Multimedia Experience (QoMEX), Lisbon, 2016, pp. 1-6. doi: 10.1109/QoMEX.2016.7498961

[16] M. Uhrina, J. Bienik, M. Vaculik and M Voznak, "Subjective video quality assessment of VP9 compression standard for full HD 
Vol.42, No.1. January2023

resolution," 2016 International Symposium on Performance Evaluation of Computer and Telecommunication Systems (SPECTS), Montreal, QC, 2016, pp. 1-5. doi: 10.1109/SPECTS.2016.7570522

[17] B. Husic', S., Barakovic', and A. Veispahic', "What factors influence the quality of experience for WebRTC video calls?" In 40th International Convention on Information and Communication Technology, Electronics and Microelectronics (MIPRO) (pp. 428-433). IEEE, May 2017.

[18] Y. Bandung, L. B. Subekti, D. Tanjung and C. Chrysostomou, "QoS analysis for WebRTC videoconference on bandwidth-limited network," 2017 20th International Symposium on Wireless Personal Multimedia Communications (WPMC), Bali, 2017, pp. 547553, doi: 10.1109/WPMC.2017.8301873.

[19] D. Vuc 'ic', and L. Skorin-Kapov, "The Impact of Packet Loss and Google Congestion Control on QoE for WebRTC-Based Mobile Multiparty Audiovisual Telemeetings," In: Kompatsiaris I., Huet B., Mezaris V., Gurrin C., Cheng WH., Vrochidis S. (eds) MultiMedia Modeling. MMM 2019. LNCS vol 11295. Springer, Cham

[20] O. Labs, "State of Video Conferencing | Owl Labs." [Online]. Available: https://www.owllabs.com/state-of-videoconferencing. [Accessed: 07-Feb-2019]. 